\title{
gp130 cytokine signaling in the pituitary gland: a paradigm for cytokine-neuro-endocrine pathways
}

\author{
Eduardo Arzt ${ }^{1,2}$ \\ ${ }^{1}$ Laboratorio de Fisiología y Biología Molecular, Facultad de Ciencias Exacias y Naturales, Universidad de Buenos Aires and Argentine \\ National Research Council (CONICET), Buenos Aires, Argentina \\ ${ }^{2}$ Max-Planck-Institute of Psychiatry, Munich, Germany \\ Address correspondence to: Eduardo Arzt, Laboratorio de Fisiología y Biología Molecular, Departamento de Biología, \\ FCEN, Universidad de Buenos Aires, Ciudad Universitaria, Pabellon II, 1428 Buenos Aires, Argentina. \\ Phone: 54-11-45763368; Fax: 54-11-45763321; E-mail: earzt@bg.fcen.uba.ar.
}

J. Clin. Invest. 108:1729-1733 (2001). DOI:10.1172/JCI200114660.

Unlike most other classes of receptors, where the cytoplasmic domain is necessary for signal transduction, receptors for IL- 6 and other related cytokines signal solely by virtue of their ability to form a larger receptor complex with a common subunit, the transmembrane glycoprotein gp130 (1). For instance, binding of IL-6 to IL-6R $\alpha$, the $\alpha$ subunit of the functional receptor, triggers the association of IL-6R $\alpha$ with gp130 $(1,2)$. Indeed, even a soluble form of IL-6R $\alpha$, entirely lacking the intracellular region of the protein, can bind to its ligand and to surface-expressed gp130, producing a normal IL-6 signal (1). The ligands of these various receptors are therefore referred to as the gp130 cytokine receptor family. As summarized in Figure 1, gp130 functions as a common cytokine signal transducer for IL-6, leukemia inhibitory factor (LIF), IL-11, oncostatin M (OSM), ciliary neurotrophic factor (CNTF), and cardiotropin-1 (CT-1), all of which bind specific receptors (their $\alpha$ subunits) but use the gp130 protein as the initial cellular signal transducer $(2,3)$. Because of this broad physiological role, the term gp130 is commonly used, in addition to its official human genome organization (HUGO) designation as the IL-6 signal transducer, or IL6ST.

The human gp130 consists of an extracellular domain of 597 amino acids, with a single transmembrane domain of 22 amino acids, and a cytoplasmic domain of 277 amino acids. As with other hemopoietic cytokine receptors, gp130 contains a cytokine receptor homology region that includes fibronectin type III domains as well as four positionally conserved cysteine residues and a WSXWS motif $(1,3)$ (Figure 1). While the expression of the gp130 cDNA alone does not confer the binding of IL-6 or the other family cytokines, gp 130 and IL-6Ra together form a high-affinity IL-6 binding site.

In the mouse, gp130 is ubiquitously expressed in adult organs, as well as in embryonic stem cells and in embryos as early as day 6 of gestation (3). Expression of gp130 therefore does not parallel that of the $\alpha$ subunit of any of the receptors of the cytokine family, nor of any specific cytokine of the IL- 6 family. These factors show some functional redundancy in the immune, hematopoietic, nervous, and neuro-endocrine systems. For example, macrophage differentiation, expression of acute-phase proteins by hepatocytes, and neuronal survival and differentiation can all be induced by multiple gp130 cytokines. Conversely, as is extensively reviewed in refs. 3-5, these cytokines also exhibit specific biological activities. Considering that gp130 is ubiquitously expressed, the time and place at which gp130 functions in vivo appears to be determined by spatially and temporally regulated expression of specific cytokine-binding receptor chains or of the cytokines themselves. In addition, soluble gp130, probably translated from an alternative spliced mRNA, can neutralize receptor signaling complexes, thereby acting as an antagonist (6). Below, I discuss the regulation of cytokine and cytokine receptor function in the pituitary gland and its importance for neuro-endocrine-stress responses.

Activation and suppression of gp130 signaling

As shown in Figure 1, association of the IL-6/IL-6R complex with gp130 leads rapidly to the homodimerization of gp 130. This homodimerization is prerequisite for signal transduction through the IL-6R complex and presumably through other related complexes involving the other receptor $\alpha$ subunits, such as the structurally similar IL-11R $(1,3)$. The IL-11 receptor complex forms a hexamer, consisting of two molecules each of IL-11, IL-11R, and gp130 (7). Likewise, LIF binds to the LIF receptor $\alpha$ subunit (LIFR $\alpha$ ), which has structural similarity to gp130 and induces the heterodimer LIFR/gp130 $(1,3)$. OSM can trigger the formation of at least two gp130 heterodimers, one with the OSM-specific receptor component OSMR $\alpha$, and the other with LIFR. CNTF binds to the CNTF receptor $\alpha$ subunit $(\mathrm{CNTFR} \alpha)$, whose extracellular regions are structurally similar to that of IL-6R $\alpha$. The resultant CNTF/CNTFR $\alpha$ complex induces the formation of the LIFR $\alpha /$ gp 130 heterodimer. Finally, CT-1 has been suggested to induce this type of heterodimer, either by direct binding to LIFR or by binding 


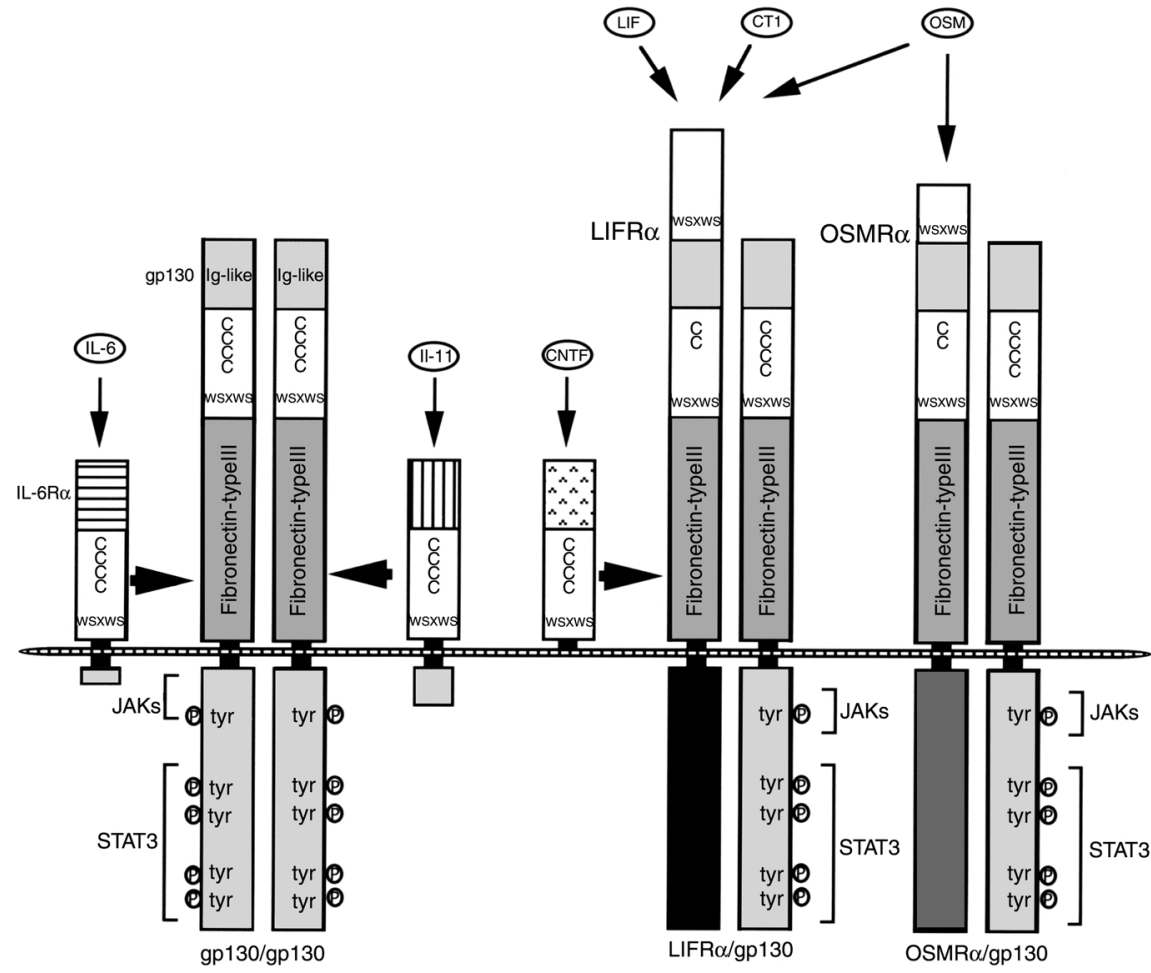

Figure 1

Schematic model of the IL-6/gp130 receptor system. The specific cytokine-binding subunits and gp130 belong to a cytokine receptor superfamily characterized by four positionally conserved cysteine residues and a WSXWS motif. Functional receptor complexes are induced by the different gp 130 cytokines: IL- 6 and IL-11 induce gp130 homodimerization; CNTF, LIF, CT-1, and OSM induce LIFR $\alpha /$ gp130 heterodimers; and OSM may also induce OSMR $\alpha / g p 130$ heterodimers. Following ligand binding, gp130 is responsible for signal transduction through the JAK/STAT pathways. to CT-1-specific receptor (CT-1R $\alpha)$ (Figure 1). As might be expected, gp130-neutralizing antibodies can inhibit the biological activities of all IL- 6 family cytokines $(1,3)$.

gp130 signals are mediated by tyrosine kinases of the JAK family and by their binding partners, transcription factors of the STAT family (reviewed in ref. 6). The general first event in activation of the JAK/STAT signaling pathway is the ligand-induced homo- or heterodimerization of signal-transducing receptor subunits - gp130 in the case of these receptors. Thereafter, JAKs (JAK1, JAK2, and Tyk2) are activated and in turn phosphorylate gp130 at several residues, providing docking sites for SH2 domain-containing molecules, such as STAT1 or STAT3 members of the STAT family. Upon phosphorylation, the STATs translocate as dimers into the nucleus, where they bind to promoter regions of their specific response genes. Altered forms of gp130 that fail to induce activation of JAKs fail to transduce an IL-6 proliferative signal. gp130 is also linked to the Ras/Raf/mitogen-activated protein kinase (Ras/Raf/MAPK) pathway via the adapter molecule SHP-2, which becomes recruited to one particular phosphotyrosine residue in the stimulated gp130 and undergoes tyrosine phosphorylation by JAKs. These cascades may activate the nuclear factor NF-IL-6, a leucine zipper-containing transcription factor homologous to C/EBP. NF-IL-6 mediates not only the expression of inflammatory cytokines, but also the expression of IL-6-inducible genes, such as acute-phase proteins in hepatocytes (3).

Activation of STATs is transient, and several mechanisms for STAT inactivation exist. First, a protein named PIAS3 blocks DNA binding of activated STAT3, as well as STAT3-mediated gene activation (6). It forms part of a group of gp130 signaling inhibitors known as protein inhibitors of activated STATs (PIAS) (6). Another mechanism to downregulate the JAK/STAT pathway consists of the suppressor of cytokine signaling (SOCS) proteins discussed by Auernhammer and Melmed in this Perspective series (ref. 8; see also ref. 6). These proteins constitute a family of negative feedback inhibitors, since their transcription is induced by IL- 6 and LIF, and they inhibit tyrosine phosphorylation of gp130, STAT1, and STAT3. SOCS-3 exerts its inhibitory function through the SHP-2 recruitment site of gp130 (9).

Gene knockouts in which such central players in IL-6 family cytokine signaling as STAT3 (10), gp130 (11), or JAK1 (12) are deleted are associated with lethal phenotypes. In keeping with the apparent redundancy of these cytokines, however, mice deficient in only one of the cytokines display only relatively mild defects.

\section{Pituitary functions of the gp130 cytokines}

The expression of gp130 mRNA in human pituitaries (13) suggests that, here as elsewhere, signaling specificity within the IL- 6 cytokine family derives from the controlled expression of the specific $\alpha$ receptor chains or of the cytokines themselves. Many expression studies have indeed demonstrated that these cytokines occur in different types of pituitary cells and could therefore act in an autocrine or paracrine manner to regulate pituitary growth and function, as well as neuro-endocrine responses.

LIF binding sites are found in the developing human fetal pituitary and in normal and adenomatous adult human tissue, as well as in murine AtT-20 corticotrophic cells (14). LIFR mRNA is induced in vivo by LPS (15). IL-6 has been extensively investigated, but only one study shows that IL- 6 receptors are expressed in rat anterior 
pituitary cells (16), and IL-6 production by anterior pituitary cells has been demonstrated by several groups (17, 18). The production of IL- 6 by these cells is increased by a variety of stimuli, such as IL-1, phorbol esters, bacterial LPS, vasoactive intestinal polypeptide (VIP), forskolin, IFNs, TNF- $\alpha$, and pituitary adenylate cyclase-activating polypeptide (PACAP) $(4,5,19)$. Interestingly, both the protein kinase $\mathrm{A}$ and the protein kinase $\mathrm{C}$ pathways appear to increase the production of IL- 6 by anterior pituitary cells, although the mechanisms involved are not yet clear. Glucocorticoids also regulate pituitary IL-6, inhibiting its production by anterior pituitary cell and aggregate cultures, as shown by the increase in pituitary levels of IL- $6 \mathrm{mRNA}$ after adrenalectomy in the rat. These data suggest that a negative feedback system links the end product of the hypothalamic-pituitary-adrenal axis (HPA) axis to IL- 6 production in the pituitary $(4,5)$. IL-6 production has been localized to folliculostellate (FS) cells of the pituitary (18), and TtT/GF, an FS cell line obtained from a pituitary thyrotropic tumor, releases IL-6 in response to VIP, PACAP, and TNF- $\alpha(4,5)$. Interestingly, LPS directly simulates IL- 6 production by FS cells via specific CD14-Toll receptors, which stimulate the $\mathrm{p} 38 \alpha$ MAPK-NF- $\mathrm{KB}$ pathway (20). Whether the other stimuli for IL- 6 act through these or other pathways remains an interesting open question.

Further evidence for IL-6 expression and regulation has been obtained in humans. IL- 6 mRNA has been detected in corticotrophic adenoma cell cultures and in normal human pituitaries and other adenoma types, such as prolactinomas, nonfunctioning adenomas, and somatotropinomas $(4,5)$. IL-1 stimulates the release of IL- 6 from cultures of human pituitary adenomas, and it has been suggested that IL- 6 expression in pituitary adenomas correlates with the aggressiveness of tumors. Consistent with the animal experiments, the synthetic glucocorticoid dexamethasone is a potent suppressor of intratumoral IL-6 production (21).

LIF is secreted by cultured bovine pituitary follicular cells (22), and the LIF protein and mRNA are found in the developing human fetal pituitary, as well as in normal and adenomatous adult human tissue (14). In pituitary explant cultures, LIF mRNA is induced by protein synthesis inhibitors (4, 5 ), suggesting that its turnover is tightly regulated. The mRNAs for murine LIF and, to a lesser extent, the LIF receptor are also induced by LPS intraperitoneal injection (15). Other gp130 cytokines and receptors also appear to function in the anterior pituitary. mRNA for IL-11 is expressed in AtT-20 corticotrophic cells (23); mRNA for CNTF is also expressed in FS and lactosomatotrophic cells (24), cell types in which the corresponding $\alpha$ chains (IL-11R and CNTFR) are also expressed $(23,24)$. IL-11R is likewise expressed in normal cells as well as in corticotrophic and nonfunctioning human tumors (23), and CNTFR has been found in tumors secreting prolactin (PRL)

Figure 2 or growth hormone $(\mathrm{GH})$ and in nonfunctioning tumors (25) and normal cells (24).

The gp130 cytokines IL-6, LIF, IL-11, and CNTF, which are thus clearly produced in the pituitary gland, act locally to influence the secretion of anterior pituitary hormones such as adrenocorticotropic hormone (ACTH), which is of particular importance for neuro-endocrine responses. Cell culture experiments with normal primary rat pituitary cells show that picomolar concentrations of IL-6 can stimulate the release of PRL, GH, and luteinizing hormone (19). IL-6 also enhances GH release from rat hemipituitary glands. The basal release of PRL is inhibited by a polyclonal antiserum to rat IL-6, a functional test showing the involvement of intrinsic IL-6 in PRL production (19). IL-6 stimulates PRL and GH release from lactosomatotrophic GH3 cells (26), and CNTF also stimulates GH and PRL production by these cells within 24 hours (24). IL-11 and CNTF exert a similar stimulation on GH mRNA expression in somatotrophic monolayer cell cultures from acromegalic tumors, and CNTF stimulates PRL secretion in lactotrophic monolayer cell cultures from prolactinoma patients (25). Interestingly, particular cell shape or cell contacts appear to be required for these signaling events. Monolayer cell cultures from normal rat anterior pituitary are largely unresponsive to IL-11 and CNTF, but when the cells are cultured as aggregates with a normal three-dimensional histological structure, both cytokines significantly stimulate secretion of PRL and GH. Thus, the three-dimensional structure of the gland, and perhaps of FS cells in particular, is of critical importance for the regulatory action of gp130 cytokines in anterior pituitary cells (25).

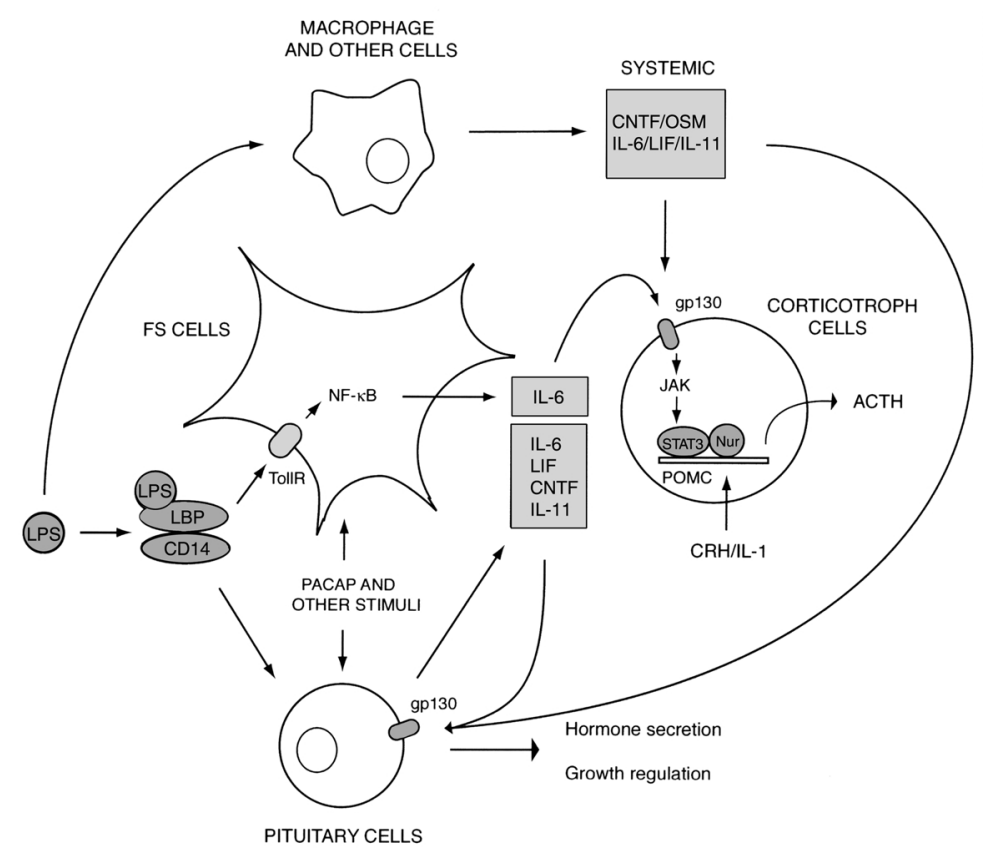

gp130 cytokine family pathways on anterior pituitary cells. Following immune (e.g., LPS) or hormonal (e.g., PACAP) stimulation, systemic or anterior pituitary gp130 cytokines, expressed by FS cells among others, induce the stimulation of ACTH (detailed in the scheme) in the pituitary. In this pathway, which is distinct from another mechanism activated by CRH or IL-1, POMC expression and ACTH secretion are stimulated through STAT3, providing a new powerful mechanism for regulation of corticotroph function. 
In addition to their effects on the secretion of pituitary hormones, gp130 cytokines can influence the growth of pituitary cells, which are normally relatively quiescent in healthy individuals. We have found that IL- 6 regulates pituitary cell growth (26). Intriguingly, in the GH3 cell line, IL-6 significantly stimulates DNA synthesis and cell number, yet the same concentrations of IL- 6 inhibit the growth of normal rat pituitary cells (26). No direct correlation between the growth effects and hormone secretion was observed in these studies. IL-6 has been also reported to stimulate growth of rat tumor pituitary cells and of $\mathrm{TtT} / \mathrm{GF}$ cells $(27,28)$. Accordingly, the latter IL-6-producing cell line stimulates growth of somatotrophic tumor cells in nude mice (29). LIF released from primary bovine pituitary cultures can also suppress the proliferation of aortic endothelial cells and is therefore considered as a factor regulating pituitary angiogenesis (22). LIF regulates proliferation of hormone-producing cells, inhibiting AtT-20 cell growth (30). IL-6 can be alternatively inhibitory or stimulatory for c-fos expression in ACTHsecreting, PRL-secreting, GH-secreting, or nonfunctioning adenomas, with no clear association between the tumor type or size and the direction of the effect. Experiments in which IL-6-blocking antibodies are introduced into the pituitary show a similarly context-dependent effect on induction of c-fos (31). Accordingly, IL-6 stimulates proliferation in some, but not all, pituitary adenomas (32). IL-11 and CNTF can also stimulate the proliferation of FS and lactosomatotrophic GH3 cells (24). Interestingly, IL-11 also stimulates the secretion of the angiogenic factor VEGF by FS cells (24).

The molecular mechanisms of gp130 control of pituitary cell proliferation are still unknown. STAT3, which is critically involved in mediating the cell growth, differentiation, and survival signals relayed through the gp130 family of cytokine receptors in immune cells (33), has not yet been shown to be directly involved in pituitary cell growth. Signaling by gp130 is considerably better characterized in the corticotrophs, as discussed below.

\section{Regulation of proopiomelanocortin and ACTH} in the neuro-endocrine-stress responses

gp130 cytokines are powerful stimuli for ACTH secretion by pituitary corticotrophs in vitro and in vivo in different species $(4,5,19)$. IL-6 stimulates the release of ACTH in normal rats, and suboptimal amounts of IL-1 and IL-6 synergize to induce an early (30-60 minute) ACTH response in mice. Consistent with this synergism, anti-IL- 6 antibody can reduce the IL-1-induced increase in plasma ACTH in mice. Studies in cell culture have provided further evidence for the stimulatory action of IL-6. This cytokine enhances ACTH release from rat hemipituitary glands and stimulates the release of ACTH from AtT-20 cells. Recombinant IL-6 has been shown to activate the HPA axis in humans, and IL-6 treatment of cancer patients induces an increase in both ACTH and cortisol plasma levels. In corticotroph adenoma cell cultures, IL- 6 also stimulates expression of proopiomelanocortin (POMC, from which ACTH is derived by posttranslational cleavage) and ACTH secretion (21), suggesting that the in vivo effect seen on corticotroph pituitary cells is direct. In mouse pituitary pri- mary cell culture, as well as in AtT-20 cells (14) and primary human fetal pituitary cultures (13), LIF, too, stimulates ACTH secretion (30). This cytokine also potentiates the stimulatory action of corticotrophin-releasing hormone $(\mathrm{CRH})$ on ACTH secretion $(13,34)$. Consistent with these cell culture data, the HPA axis is suppressed in LIF-deficient mice $(35,36)$, which show attenuated ACTH responses after immobilization stress (36). LIF knockout mice show particularly low ACTH levels following fasting; replacement of LIF restores HPA responsiveness in these mutant animals (35).

Like LIF and IL-6, OSM can stimulate ACTH in AtT-20 cells (34) and primary human fetal pituitary cultures (13). IL-11 induces POMC and ACTH secretion, as well as the expression of SOCS-3, in AtT-20 cells (23). Overexpression of SOCS-3 significantly inhibits IL-11-induced ACTH secretion (23) and also blocks the LIF-dependent activation of corticotrophs $(37,38)$, suggesting that the negative feedback system that terminates gp130 signaling in other contexts is active in these cells.

LIF rapidly induces tyrosyl phosphorylation of STAT1 and STAT3 in AtT-20 cells (34). Dominant negative forms of STAT3 block the action of LIF in the corticotroph, confirming that this mediator is required for LIF's effects (39). The LIF-responsive POMC promoter region has been mapped by deletion analysis and contains two juxtaposed sequences related to STAT3 DNAbinding motif (40). This evidence for STAT3's direct involvement offers a new mechanistic understanding of the stimulation of the HPA axis by gp130 cytokines and suggests an explanation for the synergism between CRH and LIF in regulating ACTH secretion following exposure to cytokines associated with inflammation and stress. Interestingly, this mechanism differs from the pathway by which IL-1 acts in AtT-20 corticotrophs, since neither LIF (40) nor the other gp130 cytokines (D. Kovalovsky, unpublished observations) can induce expression or activity of the transcription factors Nurr1 and Nur77 in AtT-20 cells. Hence, gp130 cytokines, in synergism with $\mathrm{CRH}$, activate the ACTH biosynthesis in the corticotrophs through a novel mechanism (41).

\section{Summary}

Specific receptors for the different gp 130 cytokines, as well as the cytokines themselves, are expressed in anterior pituitary cells, providing the basis for the regulation of hormone secretion and cell growth (Figure 2). During an inflammatory response, both IL-6 and LIF increase $(15,17)$. LPS stimulates intrapituitary IL-6 production in FS cells via specific Toll receptors using the p38 MAPK-NF-KB pathway (20). Anti-IL-6 antibodies block the ACTH response of rat anterior pituitary cell cultures to LPS, showing the involvement of locally produced IL-6 (U. Renner et al., unpublished observations). Thus, during acute or chronic inflammation or infection, systemic, hypothalamic, or hypophyseal gp130 cytokines may act on anterior pituitary cells, integrating the neuro-endocrine response.

The action of gp130 cytokines through the STAT3 transcription factor represents a powerful mechanism for regulation of pituitary corticotroph function. In response to different stressful stimuli, CRH stimulates 
the corticotrophs through cAMP/protein kinase Amediated and calcium-mediated pathways and AP-1, CREB, and Nurr transcription factors. Cytokines may act on corticotrophs through different mechanisms; whereas IL-1 acts through Nur77, gp130 employs STAT3 for transcriptional activation. Cooperation between STAT3 and other transcription factors, such as NF- $\mathrm{KB}, \mathrm{AP}-1$, or the glucocorticoid receptor, has been described in other tissues (6), but it remains to be established whether this occurs in the pituitary. Future research clarifying the molecular mechanisms of gp130 action on pituitary cells will provide new clues regarding their involvement in neuro-endocrine responses to immune stimulation and will be of great importance for understanding pituitary pathophysiology.

\section{Acknowledgments}

This work was supported by grants from the Volkswagen Foundation (I/76803), the University of Buenos Aires, the Argentine Health Ministry, the Argentine National Research Council (CONICET), and Agencia Nacional de Promoción Científica y Tecnológica-Argentina. We apologize for failing to refer, due to space constraints, to many primary sources and important references, which can be found by interested readers in the cited reviews.

1. Hirano, T. 1994. Interleukin-6. In The cytokine handbook. A.W. Thomson, editor. Academic Press Limited. London, United Kingdom. 145-168.

2. Kishimoto, T., Taga, T., and Akira, S. 1994. Cytokine signal transduction. Cell. 76:253-262.

3. Kishimoto, T., Akira, S., Narazaki, M., and Taga, T. 1995. Interleukin-6 family of cytokines and gp130. Blood. 86:1243-1254.

4. Ray, D., and Melmed, S. 1997. Pituitary cytokine and growth factor expression and action. Endocr. Rev. 18:206-228.

5. Arzt, E., et al. 1999. Pathophysiological role of the cytokine network in the anterior pituitary gland. Front. Neuroendocrinol. 20:71-95.

6. Heinrich, P.C., Behrmann, I., Muller-Newen, G., Schaper, F., and Graeve, L. 1998. Interleukin-6-type cytokine signalling through the gp130/Jak/STAT pathway. Biochem. J. 334:297-314.

7. Barton, V.A., Hall, M.A., Hudson, K.R., and Heath, J.K. 2000. Interleukin11 signals through the formation of a hexameric receptor complex. $J$. Biol. Chem. 275:36197-36203.

8. Auernhammer, C.J., and Melmed, S. 2001. The central role of SOCS-3 in integrating the neuro-immunoendocrine interface. J. Clin. Invest. 108: $1735-1740$

9. Schmitz, J., Weissenbach, M., Haan, S., Heinrich, P.C., and Schaper, F. 2000. SOCS3 exerts its inhibitory function on interleukin- 6 signal transduction through the SHP-2 recruitment site of gp130. J. Biol. Chem. 275:12848-12856.

10. Takeda, K., et al. 1997. Targeted disruption of the mouse Stat 3 gene leads to early embryonic lathality. Proc. Natl. Acad. Sci. USA. 94:3801-3804.

11. Yoshida, K., et al. 1996. Targeted disruption of gp 130, a common signal transducer for the interleukin- 6 family, leads to myocardial and hematological disorders. Proc. Natl. Acad. Sci. USA. 93:407-411.

12. Rodig, S.J., et al. 1998. Disruption of the JaK1 gene demostrates obligatory and nonredundant roles of the JaKs in cytokine-induced biologic responses. Cell. 93:373-383.

13. Shimon, I., Yan, X., Ray, D.W., and Melmed, S. 1997. Cytokine-dependent gp130 receptor subunit regulates human fetal pituitary adrenocoticotropin hormone and growth hormone secretion. J. Clin. Invest. 100:357-363.

14. Akita, S., et al. 1995. Human and murine pituitary expression of leukemia inhibitory factor. Novel intrapituitary regulation of adrenocorticotropin hormone synthesis and secretion. J. Clin. Invest. 95:1288-1298.

15. Wang, Z., Ren, S.G., and Melmed, S. 1996. Hypothalamic and pituitary leukemia inhibitory factor gene expression in vivo: a novel endotoxininducible neuro-endocrine interface. Endocrinology. 137:2947-2953.

16. Ohmichi, M., et al. 1992. Binding sites for interleukin-6 in the anterior pituitary gland. Neuroendocrinology. 55:199-203.

17. Spangelo, B.L., MacLeod, R.M., and Isakson, P.C. 1990. Production of interleukin-6 by anterior pituitary cells in vitro. Endocrinology. 126:582-586

18. Vankelecom, H., Carmeliet, P., Van Damme, J., Billiau, A., and Denef, C. 1989. Production of interleukin- 6 by folliculo-stellate cells of the anterior pituitary gland in a histiotypic cell aggregate culture system. Neuroendocrinology. 49:102-106

19. Spangelo, B.L., and Gorospe, W.C. 1995. Role of the cytokines in the neuroendocrine-immune system axis. Front. Neuroendocrinol. 16:1-12.

20. Lohrer, P., et al. 2000. Lipopolysaccharide directly stimulates the intrapituitary interleukin- 6 production by folliculostellate cells via specific receptors and the $\mathrm{p} 38 \mathrm{a}$ mitogen-activated protein kinase/nuclear factor$\kappa \mathrm{B}$ pathway. Endocrinology. 141:4457-4465.

21. Páez Pereda, M., et al. 2000. Interleukin- 6 is inhibited by glucocorticoids and stimulates ACTH secretion and POMC expression in human corticotroph pituitary adenomas. Exp. Clin. Endocrinol. Diabetes. 108:202-207.

22. Ferrara, N., Winer, J., and Henzel, W.J. 1992. Pituitary follicular cells secrete an inhibitor of aortic endothelial cell growth: identification as leukemia inhibitor factor. Proc. Natl. Acad. Sci. USA. 89:698-702.

23. Auernhammer, C.J., and Melmed, S. 1999. Interleukin-11 stimulates proopiomelanocortin gene expression and adrenocorticotropin secretion in corticotroph cells: evidence for a redundant cytokine network in the hypothalamo-pituitary-adrenal axis. Endocrinology. 140:1559-1566.

24. Perez Castro, C., et al. 2000. The gp130 cytokines interleukin-11 and ciliary neurotropic factor regulate through specific receptors the function and growth of lactosomatotropic and folliculostellate pituitary cell lines. Endocrinology. 141:1746-1753.

25. Perez Castro, C., et al. 2001. Effects of the gp130 cytokines CNTF and IL-11 on pituitary cells: CNTF receptors on human pituitary adenomas and stimulation of PRL and GH secretion in normal rat anterior pituitary aggregate cultures. J. Endocrinol. 169:539-547.

26. Arzt, E., et al. 1993. Interleukin involvement in anterior pituitary cell growth regulation: effects of interleukin-2 (IL-2) and IL-6. Endocrinology. 132:459-467.

27. Renner, U., Gloddek, J., Arzt, E., Inoue, K., and Stalla, G.K. 1997. Interleukin-6 is an autocrine growth factor for TTT/GF mouse pituitary cells. Exp. Clin. Endocrinol. Diabetes. 105:345-352.

28. Sawada, T., et al. 1995. Interleukin-6 stimulates cell proliferation of rat anterior pituitary clonal cell lines in vitro. J. Endocrinol. Invest. 18:83-90.

29. Koyama, C., et al. 1995. Pituitary folliculo-stellate-like cells stimulate somatotropic pituitary tumor growth in nude mice. Endocr. Pathol. 6:67-75.

30. Stefana, B., Ray, D.W., and Melmed, S. 1996. Leukemia inhibitory factor (LIF) induces differentiation of pituitary corticotroph function: a neuroendocrine phenotypic switch. Proc. Natl. Acad. Sci. USA. 93:12502-12506.

31. Páez Pereda, M., et al. 1996. Interleukin-2 (IL-2) and IL-6 regulate c-fos protooncogene expression in human pituitary adenoma explants. Mol. Cell. Endocrinol. 124:33-42.

32. Jones, T.H., and Justice, S.K. 1995. Effect of interleukin-6 on human pituitary tumor cell growth. J. Endocrinol. 144:P292. (Abstr.)

33. Hirano, T., Ishihara, K., and Hibi, M. 2000. Roles of STAT3 in mediating the cell growth, differentiation and survival signals relayed through the IL-6 family of cytokine receptors. Oncogene. 19:2548-2556.

34. Ray, D.W., Ren, S.G., and Melmed, S. 1996. Leukemia inhibitory factor (LIF) stimulates proopiomelanocortin (POMC) expression in a corticotroph cell line. Role of STAT pathway. J. Clin. Invest. 97:1852-1859.

35. Akita, S., Malkin, J., and Melmed, S. 1996. Disrupted murine leukemia inhibitory factor (LIF) gene attenuates adrenocorticotropic hormone (ACTH) secretion. Endocrinology. 137:3140-3143.

36. Chesnokova, V., Auerhamer, C.J., and Melmed, S. 1998. Murine LIF gene disruption attenuates the hypothalamio-pituitary-adrenal axis stress response. Endocrinology. 139:2209-2216.

37. Bousquet, C., Susini, C., and Melmed, S. 1999. Inhibitory roles for SHP1 and SOCS-3 following pituitary proopiomelanocortin by leukemia inhibitory factor. J. Clin. Invest. 104:1277-1285.

38. Auernhammer, C.J., Chesnokova, V., Bousquet, C., and Melmed, S. 1998. Pituitary corticotroph SOCS-3: novel intracellular regulation of leukemia-inhibitory factor-mediated proopiomelanocortin gene expression and adrenocorticotropin secretion. Mol. Endocrinol. 12:954-961.

39. Bousquet, C., and Melmed, S. 1999. Critical role for STAT3 in murine pituitary adrenocorticotropin hormone leukemia inhibitory factor signaling. J. Biol. Chem. 274:10723-10730.

40. Bousquet, C., Zatelli, M.C., and Melmed, S. 2000. Direct regulation of pituitary proopiomelanocortin by STAT3 provides a novel mechanism for immuno-nueroendocrine interfacing. J. Clin. Invest. 106:1417-1425.

41. Bousquet, C., Chesnokova, V., Kariagina, A., Ferrad, A., and Melmed, S. 2001. cAMP neuropeptide agonists induce pituitary suppressor of cytokine signaling-3: novel negative feedback mechanism for corticotroph cytokine action. Mol. Endocrinol. 15:1880-1890. 